\title{
Predição do Apego de Crianças em Função do Estilo Educativo Materno e do Tipo de Família
}

\author{
Prediction of Children's Attachment Regarding Maternal \\ Rearing Style and Family Type
}

\author{
Sónia Catarina Carvalho Simões ${ }^{*}, a, b$, Carlos Farate $^{a, b}$, Isabel Soares $^{c} \&$ João Duarte ${ }^{d}$ \\ ${ }^{a}$ Instituto Superior Miguel Torga, Coimbra, Portugal \\ ${ }^{b}$ Instituto de Ciências Biomédicas Abel Salazar, Universidade do Porto, Porto, Portugal \\ ${ }^{c}$ Escola de Psicologia, Universidade do Minho, Braga, Portugal. \\ $\&{ }^{d}$ Escola Superior de Saúde de Viseu, Viseu, Portugal.
}

\begin{abstract}
Resumo
Este estudo teve como objetivo identificar fatores preditores da segurança do apego das crianças. O estudo foi conduzido numa amostra comunitária de mães de 288 crianças em idade escolar, distribuídas por três grupos de acordo com a tipologia familiar (famílias nucleares, monoparentais e reconstituídas). As mães avaliaram o seu estilo educativo (EMBU-P) e os comportamentos de apego dos filhos (PCV-M). Os resultados evidenciaram uma correlação positiva entre as perceções maternas dos comportamentos de apego dos filhos e de suporte emocional e uma correlação negativa entre esta mesma variável e a rejeição materna. O modelo preditor da segurança do apego identificou como preditores a rejeição e o suporte emocional maternos, não se verificando o poder preditivo do tipo de família.

Palavras-chave: Criança, segurança do apego, segurança da vinculação, estilo educativo materno; tipo de família.

Abstract

This study aims to identify predictive factors of children's secure attachment. The study was conducted in a community-based sample of 288 mothers of school-aged children distributed in three groups accordingly to their family type (nuclear, single-parent and reconstituted family). The mothers assessed their own rearing style (EMBU-P) as well as their children's attachment behaviors (PCV-M). The results showed a positive correlation between mothers' perception of emotional support and the quality of their children's attachment behaviors, and a negative correlation between the latter variable and maternal rejection. The predictive model of attachment security identified maternal rejection and emotional support as predictors, whilst family type showed no predictive power.

Keywords: Children, attachment security, maternal rearing style, family type.
\end{abstract}

$\mathrm{Na}$ literatura podemos encontrar diversas abordagens da parentalidade, que realçam diferentes construtos teóricos. Algumas teorias da parentalidade abordam o processo de socialização da criança. Uma das áreas mais desenvolvidas da parentalidade é o estudo dos estilos educativos parentais, que engloba a relação emocional estabelecida entre pais e filhos, os sistemas de crenças, as práticas educativas e os comportamentos dos pais (Darling \& Steinberg, 1993; Dishion \& McMahon, 1998). Segundo esta abordagem o comportamento parental deve contemplar duas dimensões: (a) de natureza afetiva, caracterizada pela presença de responsividade, suporte e afeto parental, e (b) de tipo instrumental, na qual o con-

\footnotetext{
*Endereço para correspondência: Departamento de Investigação e Desenvolvimento, Instituto Superior Miguel Torga, Largo da Cruz de Celas, 1, Coimbra, Portugal 3000-132. E-mail: soniasimoes76@gmail.com
}

trolo e a disciplina são usados com o objetivo de socializar a criança, ao promover o cumprimento das regras e normas sociais (Darling \& Steinberg, 1993).

Outras teorias da parentalidade focam-se, primariamente, no contexto emocional da relação pais-filho, como a teoria do apego ao defender que os pais que são cuidadores emocionalmente apoiantes, afetuosos, sensíveis e responsivos promovem um apego seguro com os seus filhos, o que tem consequências desenvolvimentais positivas (Cassidy, 1999). De acordo com a teoria do apego desenvolvida por Bowlby (1969/1984), há uma predisposição para a criança procurar e manter a proximidade com uma figura privilegiada, denominada de figura de apego, que assegura a sua segurança e proteção. O processo de apego estabelece-se durante o primeiro ano de vida do bebé, pelo desenvolvimento de um vínculo privilegiado com a figura que lhe presta cuidados. $\mathrm{O}$ apego da criança pode ser definido como um laço afetivo, que se prolonga no 
tempo, que a criança estabelece com a figura de apego através das interações no quotidiano. Deste modo, nos primeiros anos de vida, através das interações que estabelece com os seus cuidadores primários, a criança cria expetativas sobre a sua disponibilidade e responsividade, que servem como base para a elaboração do modelo interno dinâmico. $\mathrm{O}$ modelo interno dinâmico remete para representações mentais sobre os outros, sobre o mundo e sobre o Self, em termos de ser, ou não, merecedor de afeto e proteção, sendo usado como protótipo das relações que o sujeito estabelece ao longo da vida (Bowlby, 1969/ 1984; Kerns, Schlegelmilch, Morgan, \& Abraham, 2005; Soares, 2007).

As primeiras investigações sobre o apego dedicaramse ao estudo da primeira infância, mas a teoria do apego tem alargado o seu estudo a outras fases do desenvolvimento. Não obstante, a avaliação do apego de crianças em idade escolar está em fase embrionária, sendo reconhecida a necessidade de avaliar o desenvolvimento do apego durante este período do ciclo vital (Kerns et al., 2005).

O estudo do apego deve contemplar diversas variáveis individuais, relacionais e contextuais que podem influenciar de modo bidirecional o relacionamento da criança com cada um dos progenitores. Nesta linha, a organização do apego é influenciada tanto pelas caraterísticas individuais da criança e da figura de apego, como pelo comportamento parental (e.g. atitudes, valores, motivação para a parentalidade, suporte/afeto parental, responsividade contingente, sensibilidade, grau de investimento no filho), e pelos fatores contextuais (suporte social, nível socioeconómico, presença de estressores familiares, tipo de família e influências socioculturais diversas), não esquecendo que o próprio sistema pode influenciar o contexto (Thompson \& Raikes, 2003; van IJzendoorn \& SagiSchwartz, 2008).

No que respeita ao impacto das figuras parentais na segurança do apego da criança, a teoria do apego tem-se debruçado principalmente sobre o estudo da sensibilidade materna. Contudo, as associações modestas entre o apego da criança e a sensibilidade materna sugerem que, embora seja um fator determinante para a qualidade do apego da criança, não é o mecanismo de explicação exclusivo. É, então, reconhecida a importância da análise da influência de outras variáveis individuais e contextuais na qualidade do apego e na transmissão intergeracional do apego (Thompson \& Raikes, 2003; van IJzendoorn \& Sagi-Schwartz, 2008), nomeadamente as práticas educativas específicas e os estilos educativos que os pais utilizam na educação dos filhos. As variáveis enumeradas podem ajudar a explicar a qualidade do apego da criança, bem como algumas das associações entre apego e desenvolvimento infantil.

São poucas as pesquisas sobre a relação específica entre a qualidade do apego da criança e os estilos educativos parentais. Muitos dos estudos sobre a relação entre os estilos educativos parentais e a qualidade do apego da criança são de natureza correlacional, pelo que não permitem elucidar de modo absoluto sobre o sentido das influências (Muris, Meesters, Merckelbach, \& Hülsenbeck, 2000). Assim, apesar de ter sido evidenciada a associação entre as dimensões do comportamento parental e a qualidade do apego (Nair \& Murray, 2005), não se pode esquecer a bidirecionalidade das interações entre as dimensões do comportamento parental e a qualidade do apego da criança.

No que respeita ao modo como as diferentes dimensões dos estilos educativos parentais influenciam a qualidade do apego de crianças em idade escolar, a investigação tem evidenciado o suporte emocional e a rejeição como as dimensões que mais contribuem, de modo positivo e negativo respectivamente, para a qualidade do apego das crianças (e.g. Brown \& Whiteside, 2008; Karavasilis, Doyle, \& Markiewicz, 2003; Roelofs, Meesters, \& Muris, 2008; Roelofs, Meesters, ter Huurne, Bamelis, \& Muris, 2006). Mais especificamente, os estudos que utilizam o Egna Minnen Besträffende Uppfostran-P (EMBU-P; Castro, Pablo, Gómez, Arrindel, \& Toro, 1997), instrumento usado no presente estudo para avaliar o estilo educativo materno, têm descrito uma associação entre apego inseguro e níveis mais elevados de rejeição parental e, em oposição, entre apego inseguro e níveis mais baixos de suporte/afeto parental (Muris et al., 2000; Muris, Meesters, \& van der Berg, 2003; Roelofs et al., 2008). Assim, estilos educativos parentais caracterizados por elevados níveis de rejeição e baixos níveis de suporte emocional estão relacionados com uma menor qualidade do apego das crianças, ao passo que uma menor rejeição e um maior suporte emocional promovem um apego seguro.

Por conseguinte, a investigação indica, genericamente, que os estilos educativos parentais de suporte, afeto e aceitação estão relacionados com o estabelecimento de um apego seguro na criança (Kerns, Aspelmeier, Gentzler, \& Grabill, 2001; Michiels, Grietens, Onghena, \& Kuppens, 2010; Roskam, Henry, Collin, \& Manil, 2008). Nesta linha, tem sido confirmada a importância do comportamento parental para a segurança do apego da criança, nomeadamente a presença de apoio emocional e sensibilidade, com níveis adequados de controlo positivo e baixos níveis de rejeição (Allen et al., 2003; de Minzi, 2006; Kerns et al., 2001). Os resultados parecem convergir com a importância dada pela teoria do apego à qualidade dos cuidados parentais, reforçando a ideia de que, para além da sensibilidade materna, também o estilo educativo materno, nomeadamente a dimensão suporte/ rejeição, poderá ter um grande impacto na qualidade do apego das crianças, uma vez que nos endereça para a qualidade da relação entre pais e filhos. Neste contexto, pode colocar-se como hipótese que são as dimensões afetivas do comportamento materno (suporte emocional e rejeição) as que têm uma maior influência na qualidade do apego da criança. 
A atualidade é marcada pelo aumento da taxa de divórcios e do número de famílias monoparentais e reconstituídas. A literatura tem descrito que os comportamentos parentais podem ser influenciados negativamente pelos fatores de estresse a que os pais estão sujeitos (e.g. monoparentalidade, luto, conflito conjugal, divórcio). Neste sentido, o divórcio tem sido associado a alterações na qualidade da relação dos pais com a criança e a dificuldades de ajustamento. Esta alteração na estrutura familiar pode influenciar os comportamentos parentais, ao deixar os pais menos disponíveis física e emocionalmente para os seus filhos, principalmente durante os primeiros anos após a separação. Os estudos têm, ainda, indicado que as mães divorciadas aplicam práticas parentais mais disfuncionais, utilizam menos a monitorização, são menos afetuosas e mais críticas (Hetherington, Bridges, \& Insabella, 1998; Nair \& Murray, 2005).

Acrescente-se que as pesquisas descrevem a monoparentalidade como uma realidade que cria condições desfavoráveis à parentalidade funcional e saudável, principalmente devido à sua frequente associação a fatores de risco. Isto é, os pais sozinhos têm menor probabilidade de beneficiar de suporte social, maior probabilidade de passar por dificuldades económicas e acontecimentos de vida estressantes, de sofrer de depressão, nomeadamente devido a pressões económicas e por acumular as funções que são partilhadas por ambos os elementos do casal numa família nuclear (Kalenkoski, Ribar, \& Statton, 2005).

Vários estudos longitudinais têm identificado os fatores de risco que afetam o desenvolvimento sócioemocional das crianças em idade escolar, entre eles a separação (K. E. Grossmann, K. Grossmann, \& Kindler, 2005; Hetherington et al., 1998) e a rejeição parental (K. E. Grossmann et al., 2005; Pettit, Bates, \& Dodge, 1997; Trautmann-Villalba, Gschwendt, Schmidt, \& Laucht, 2006). Boa parte dos estudos que comparam diferentes estruturas familiares descreve uma associação entre a exposição da criança a fatores de estresse, como a separação dos pais, e o empobrecimento da qualidade do apego da criança (Moura \& Matos, 2008; Tippelt \& Konig, 2007; Woodward, Fergusson, \& Belsky, 2000), bem como perceções mais negativas sobre os cuidados parentais (Kilmann, Carranza, \& Vendemia, 2006; Schwartz \& Finley, 2009; Woodward et al., 2000). Assim, tem sido referido que os pais em famílias monoparentais e reconstituídas utilizam estilos educativos menos positivos, em comparação com os pais de famílias nucleares (Kalenkoski et al., 2005; Kelly, 2007; Lundberg \& Andersson, 2000). No mesmo sentido, as crianças de famílias monoparentais e reconstituídas têm um percurso desenvolvimental mais desfavorável, nomeadamente ao nível da qualidade do apego (Faber \& Wittenborn, 2010; Nair \& Murray, 2005; Tippelt \& Konig, 2007).

Porém, tem sido documentado que, para além do tipo de família, muitos outros fatores podem influenciar o comportamento parental e a qualidade do apego das crianças, interferindo nesta leitura aparentemente linear das relações entre variáveis. De fato, estas dimensões da interação pais-filhos podem ser afetadas por fatores como a qualidade da relação entre os pais e a qualidade da coparentalidade (Feinberg \& Kan, 2008; Kaczynski, Lindahl, Malik, \& Laurenceau, 2006).

Não obstante, são poucos os estudos que analisam a influência do tipo de família tanto nos estilos educativos parentais, como na qualidade do apego de crianças durante o período escolar. Deste modo, o presente estudo tem o propósito de contribuir para enriquecer o conhecimento neste domínio, partindo do pressuposto de que a segurança do apego da criança é influenciada pelo estilo educativo materno. Em linha com a revisão teórica efetuada, são objetivos deste estudo empírico: (a) avaliar a relação entre as perceções maternas dos estilos educativos e da qualidade do comportamento de apego dos filhos em idade escolar; (b) Verificar um modelo preditor da influência que o tipo de família e as dimensões do estilo educativo materno exercem sobre a segurança do comportamento de apego das crianças.

\section{Método}

\section{Participantes}

Este estudo inclui 288 mães de crianças em idade escolar, distribuídas por três grupos de acordo com a tipologia familiar quanto à sua composição: o grupo 1 é constituído por 116 díades mãe-filho de famílias nucleares, em que as crianças vivem com ambos os pais; o grupo 2 é constituído por 116 díades que provêm de famílias monoparentais, em que a criança vive apenas com a mãe; o grupo 3 é composto pelas restantes 56 díades pertencentes a famílias reconstituídas, vivendo a criança com a mãe e com o padrasto.

A comparação dos três grupos em função das variáveis sociodemográficas (idade e género da criança, número de irmãos, idade e habilitações literárias da mãe) não evidenciou diferenças estatísticas significativas entre os três grupos de famílias.

As crianças frequentam o $3^{\circ}$ ano $(34,0 \%)$ e o $4^{\circ}$ ano $(66,0 \%)$ de escolaridade do $1^{\circ}$ ciclo do ensino básico, têm idades compreendidas entre os 7 e os 11 anos $(M=8,88)$, sendo $51,7 \%$ da amostra constituída por meninas. É mais frequente a criança ser filha única $(45,1 \%)$, seguindo-se as crianças com um irmão $(40,7 \%)$, sendo menos frequente que a criança tenha 2 ou mais irmãos $(14,2 \%)$.

A idade das mães variou entre os 23 e os 53 anos $(M=36,82 ; D P=5,35)$. No que respeita às habilitações literárias das mães, a maioria completou 10 anos de escolaridade ou mais $(59,0 \%)$ e apenas uma minoria tem 4 anos de escolaridade (5,9\%). As subamostras do estudo distribuem-se quase equitativamente pelas zonas de residência rural e urbana. A esmagadora maioria das crianças do estudo é caucasiana $(96,9 \%)$. 
Simões, S. C. C., Farate, C., Soares, I. \& Duarte, J. (2013). Predição do Apego de Crianças em Função do Estilo Educativo Materno e do Tipo de Família.

\section{Instrumentos}

Caraterização da Criança e do Contexto Familiar. Com o objetivo de obter uma caraterização da amostra em estudo foi utilizado um questionário psicossocial (adaptado de Farate, Pocinho, \& Machado, 2010) composto por um conjunto de questões fechadas e pré-codificadas, que teve como objetivo fazer uma caracterização sociodemográfica dos pais e da criança e recolha de informação sobre a saúde e o desenvolvimento da criança.

Uma primeira secção é composta pelas seguintes variáveis: idade; nível de escolaridade, situação profissional e profissão do pai e da mãe; situação conjugal atual dos pais; número de filhos, idade e género; composição do agregado familiar. Posteriormente, são contempladas questões retrospetivas sobre o filho, desde a gravidez e parto até ao momento atual. São apresentadas questões sobre a saúde da criança, incluindo o sono, a alimentação e o desenvolvimento psicomotor e afetivo. Segue-se o questionamento sobre as variáveis comportamentais, o modo de guarda da criança, a adaptação escolar desde o infantário, o comportamento e o rendimento escolares. Por fim, completam o questionário um ponto sobre os acontecimentos de vida da criança desde o nascimento e questões sobre a qualidade da relação da mãe com o pai do seu filho e a frequência com que o pai vê o filho.

Perceção Materna do Estilo Educativo Materno. A perceção que a mãe tem do seu estilo educativo foi avaliada através do Egna Minnen Besträffende Uppfostran-P (EMBU-P; Castro et al., 1997; versão portuguesa de Canavarro \& Pereira, 2007). Este instrumento psicométrico foi desenvolvido a partir do original EMBU de C. Perris, Jacobsson, Lindstrom, von Knorring e H. Perris (1980), que avalia as memórias dos adultos sobre o comportamento parental dos seus pais.

A versão portuguesa do EMBU-P é composta por $3 \mathrm{fa}-$ tores (suporte emocional, rejeição e tentativa de controlo), dispostos em 42 itens organizados em escala ordinal tipo Likert com quatro alternativas de resposta (1: "não, nunca"; 2: "sim, às vezes"; 3 : "sim, frequentemente"; 4 : "sim, sempre"), sendo pedido à mãe que escolha a resposta que melhor reflita o comportamento que teve ou tem para com o seu filho.

A escala suporte emocional destina-se a avaliar os estilos educativos caraterizados pela expressão verbal e física de suporte afetivo dos pais, a aceitação materna e a disponibilidade física e psicológica. A escala rejeição contempla os itens que avaliam a manifestação de hostilidade/agressão verbal e física da mãe e não-aceitação da criança. A escala tentativa de controlo reúne os itens em que as mães empreendem tentativas de controlar o comportamento dos filhos, demonstrações de exigência e de grande preocupação em relação ao bem-estar dos filhos (Canavarro \& Pereira, 2007).

Os coeficientes de alpha de Cronbach apresentam valores razoáveis a bons de consistência interna no EMBU$P$ respondido pelas mães: 0,82 na escala suporte emocio- nal, 0,87 na escala rejeição e 0,73 na escala tentativa de controlo (Canavarro \& Pereira, 2007).

No presente estudo o EMBU-P mostrou valores de alpha de Cronbach adequados, próximos dos indicados pelos autores, isto é, de $0,798,0,723$ e 0,703 , respetivamente, para as escalas suporte emocional, rejeição e tentativa de controle. Assim, a consistência interna do EMBU-P oscila entre o razoável e o bom.

Qualidade do Comportamento de Apego da Criança. A avaliação da perceção da mãe relativamente aos comportamentos de apego do seu filho no período escolar foi realizada com recurso à Escala de Perceção Materna do Comportamento de Vinculação da Criança (PCV-M de Dias, Soares, \& Freire, 2002). Este instrumento baseiase na opinião da mãe acerca de um conjunto de comportamentos que o filho pode ter apresentado ou apresenta na relação que estabelece com os pais, sendo constituído por 33 itens cotados numa escala tipo Likert de 5 pontos, em que 1 significa "totalmente diferente do meu filho(a)" e 5 "totalmente parecido com o meu filho(a)".

O PCV-M contempla três dimensões importantes do comportamento de apego de crianças em idades escolar: autorregulação emocional, partilha de afeto, comportamento base-segura, bem como inclui a dimensão desejabilidade social das mães (Dias et al., 2002). Considera-se que o funcionamento apropriado da criança nestas dimensões resulta de uma relação de apego seguro (Dias et al., 2002).

A dimensão dificuldades de autorregulação emocional avalia indicadores de insegurança da relação de apego quando o sistema de apego da criança é ativado; a dimensão comportamento base-segura permite avaliar os comportamentos indicadores de utilização das figuras de apego como base-segura para a exploração; a partilha de afeto é a dimensão que reporta os comportamentos de partilha de experiências e de afeto com as figuras de apego; por fim, a dimensão desejabilidade social descreve itens com pouca probabilidade de serem observados nesta idade, com o objetivo de identificar valores de desejabilidade social que possam comprometer a validade das respostas dos pais (Dias et al., 2002). A pontuação global do PCV-M é um indicador da segurança do apego da criança, resultando do somatório das 4 subescalas, em que a dimensão dificuldades de autorregulação emocional é contabilizada com os itens invertidos. Esta subescala é cotada de forma invertida porque mede as dificuldades da criança em regular as suas emoções e interessa calcular um indicador da segurança do apego (Dias et al., 2002).

No estudo preliminar da escala (Dias et al., 2002), a consistência interna do PCV-M revela os valores de alpha de Cronbach de 0,88, 0,82, 0,76, e 0,86, respetivamente nas dimensões dificuldades de autorregulação emocional (DARE), comportamento base segura (CBS), partilha de afeto (PA) e PCV-M global. O nível de consistência interna da dimensão desejabilidade social não foi avaliado 
no estudo de construção da escala, pois esta subescala foi incluída aquando do desenvolvimento da prova, com o único intuito de identificar casos extremos de desejabilidade social das mães, denotando enviesamento nas respostas dadas.

Igualmente, o presente estudo revelou bons índices de consistência interna, com valores para o alpha de Cronbach de $0,829,0,770,0,769,0,758$ e 0,859 , respetivamente, nas dimensões dificuldades de autorregulação emocional, comportamento base-segura, partilha de afeto, desejabilidade social e escala global, valores que podem ser classificados como bons.

\section{Resultados}

Relação entre a Perceção Materna do Comportamento de Apego e a Perceção Materna do Estilo Educativo

O suporte emocional e a rejeição do EMBU-P são as dimensões da perceção materna do estilo educativo que evidenciam uma correlação mais significativa com a perceção materna do comportamento de apego dos filhos, já que apresentam correlações com as quatro dimensões do PCV-M e com a pontuação global da escala. Como se pode ver na Tabela 1, os resultados indicam que, no que diz respeito à correlação entre estilos educativos parentais e comportamento de apego das crianças, as mães que providenciam maior suporte emocional aos seus filhos, identificam nos seus filhos um comportamento de apego mais seguro, visto que se encontra uma correlação positiva significativa entre a dimensão suporte emocional do EMBU-P e a pontuação global do PCV-M $(r=0,320$, $p<0,01$ ), assim como com cada uma das dimensões do PCV-M, consideradas separadamente. Neste sentido, a dimensão suporte emocional do EMBU-P apresenta uma correlação positiva com as subescalas comporta- mento base-segura $(r=0,329, p<0,01)$ e partilha de afeto $(r=0,236, p<0,01)$ e negativa com a subescala dificuldades de autorregulação emocional $(r=-0,219, p<0,01)$ do PCV-M. Pode então dizer-se que, à perceção das mães de que prestam um bom suporte emocional, correspondem os filhos com menores dificuldades de autorregulação emocional, mais comportamentos de base-segura, maior partilha de afeto e, em síntese, com um comportamento de apego mais seguro.

Por outro lado, as mães que percecionam mais comportamentos de rejeição para com os seus filhos consideram que eles têm um comportamento de apego mais inseguro, na medida em que se encontra uma correlação negativa significativa da dimensão rejeição do EMBU-P com a pontuação global do PCV-M $(r=-0,388, p<0,01)$, bem como com cada uma das dimensões do PCV-M consideradas isoladamente. Assim, a dimensão rejeição do EMBU-P apresenta uma correlação negativa com as subescalas do PCV-M comportamento base-segura ( $r=-$ $0,271, p<0,01)$ e partilha de afeto $(r=-0,181, p<0,01)$ e uma correlação positiva com a subescala dificuldades de autorregulação emocional $(r=0,331, p<0,01)$. Isto quer dizer que as mães que referem comportamentos de rejeição relativamente aos seus filhos identificam-lhes um comportamento de apego mais inseguro, isto é, menos comportamentos de base-segura, menor capacidade para partilhar afetos e maiores dificuldades de autorregulação emocional.

Já a dimensão tentativa de controlo do EMBU-P apenas se correlaciona de modo significativo com a autorregulação emocional avaliada pelo PCV-M $(r=0,172$, $p<0,01)$, ou seja, as mães que consideram ter mais comportamentos de controlo em relação aos seus filhos admitem que estes têm maiores dificuldades de autorregulação emocional.

Tabela 1

Correlação entre a Perceção Materna do Comportamento de Apego e do Estilo Educativo

\begin{tabular}{lccccc}
\hline \multicolumn{7}{c}{ PCV-M } & \multicolumn{1}{l}{} \\
\hline EMBU-P & DARE & CBS & PA & DS & \multicolumn{1}{c}{ Global } \\
\hline Suporte emocional & $-0,219 * *$ & $0,329 * *$ & $0,236 * *$ & $0,180 * *$ & $0,320 * *$ \\
Rejeição & $0,331^{* *}$ & $-0,271^{* *}$ & $-0,181 * *$ & $-0,328 * *$ & $-0,388^{* *}$ \\
Tentativa controlo & $0,172 * *$ & $-0,027$ & 0,044 & 0,030 & $-0,072$ \\
\hline
\end{tabular}

${ }^{*} p<0,050 ; * *^{*}<0,010$ (Coeficiente de Pearson).

Predição da Segurança do Comportamento de Apego. A fim de testar um modelo preditor da segurança do comportamento de apego da criança para o nosso estudo empírico, foram realizadas análises de regressão linear pelo método stepwise. Como variável dependente foi considerada a pontuação global do PCV-M, que é um indicador da perceção materna acerca da segurança do apego da criança. De modo a que a variável tipo de família pudesse integrar o modelo de regressão, foi transformada em variável "muda". Assim, foram selecionadas como variáveis preditivas da segurança do apego da criança as dimensões do EMBU-P e o tipo de família.

A Tabela 2 reporta os resultados obtidos no último modelo, evidenciando duas variáveis que se constituíram como preditoras da segurança do apego da criança: as perceções maternas de rejeição e de suporte emocional. Todas as outras variáveis foram excluídas do modelo, incluindo o tipo de família de pertença. 
A variável rejeição materna foi a primeira variável a entrar no modelo de regressão, que configurou dois passos, explicando no primeiro passo $11,6 \%$ da variação do indicador de segurança do apego da criança, com um erro padrão de regressão de 17,479. No modelo final é acrescentada a dimensão suporte emocional materno, sobressaindo a correlação moderada $(r=0,440)$ que o conjunto destas variáveis estabelece com a pontuação global do PCV-M. No conjunto, o modelo explica $18,2 \%$ da variação deste indicador da segurança do apego da criança, baixando o erro padrão de estimativa para 16,817 .

As variáveis presentes no modelo não são colineares, dado que os valores da VIF são de 1,022 na rejeição e no suporte emocional maternos. Por outro lado, os testes $F$ e os valores de $t$, ao apresentarem-se estatisticamente significativos, permitem afirmar que as variáveis que entraram no modelo têm valor explicativo da variável dependente pontuação global do PCV-M. Os coeficientes padronizados beta indicam que a rejeição materna é a variável que se apresenta com maior peso preditor, seguida do suporte emocional materno, apresentando a primeira variável uma relação inversa e a segunda uma relação direta com o indicador da segurança do comportamento de apego, resultante do cálculo da pontuação global do PCV-M. Assim, pode ser inferido que uma perceção materna de menor rejeição e de maior suporte emocional corresponde a uma maior segurança do comportamento de apego da criança.

Por fim, a seguinte fórmula resume o modelo final ajustado para o indicador segurança do apego, calculado a partir da pontuação global do PCV-M: Indicador da segurança do apego da criança $=113,982+(-0,1.344$ rejeição materna $)+(1,047$ suporte emocional materno $)$

Tabela 2

Regressão Múltipla entre o PCV-M Global e as Variáveis Independentes

\begin{tabular}{|c|c|c|c|c|c|c|}
\hline Variável Dependente & $\begin{array}{c}R \\
\left(R^{2} \text { ajustado }\right)\end{array}$ & $\begin{array}{c}R^{2} \\
\text { estimativa }\end{array}$ & Erro padrão & Incremento $R^{2}$ & $F$ & $p$ \\
\hline PCV-M global & 0,440 & $0,193(0,182)$ & 16,817 & 0,071 & 12,393 & 0,001 \\
\hline \multicolumn{7}{|l|}{ Pesos de regressão } \\
\hline $\begin{array}{l}\text { Variáveis } \\
\text { independentes }\end{array}$ & $\begin{array}{l}\text { Coeficiente } \\
\text { beta }\end{array}$ & $\begin{array}{l}\text { Coeficiente } \\
\text { Padronizado }\end{array}$ & $T$ & $p$ & Colinearidade VIF & \\
\hline Constante & 113,982 & - & - & - & - & \\
\hline Rejeição EMBU-P & $-1,344$ & $-0,311$ & $-4,064$ & 0,000 & 1,022 & \\
\hline S. Emocional EMBU-P & 1,047 & 0,269 & 3,520 & 0,001 & 1,022 & \\
\hline \multicolumn{7}{|l|}{ Análise de variância } \\
\hline Efeito & Soma quadrados & $g l$ & Média quadrados & $F$ & $p$ & \\
\hline Regressão & 9563,427 & 2 & 4781,714 & 16,908 & 0,000 & \\
\hline Residual & 39876,010 & 141 & 282,809 & - & - & \\
\hline Total & 49439,438 & 143 & - & - & - & \\
\hline
\end{tabular}

\section{Discussão e Conclusões}

Os resultados revelam correlações entre as diferentes dimensões do estilo educativo materno e o comportamento de apego das crianças. No estudo da relação entre o comportamento de apego da criança e o estilo educativo materno, os resultados evidenciam uma convergência entre a qualidade do comportamento de apego e a dimensão suporte emocional do EMBU-P, pelo que se constata que o suporte emocional da mãe parece ser essencial para o apego seguro do seu filho. Assim, as mães que providenciam maior suporte emocional aos seus filhos identificam neles um comportamento de apego mais seguro.

Estes resultados estão de acordo com os estudos que têm identificado a dimensão suporte emocional como tendo um impato positivo no desenvolvimento da criança e como estando associada positivamente à segurança do apego da criança (Baumrind, 1989; Dishion \& McMahon, 1998; Kerns et al., 2001; Michiels et al., 2010; Roskam et al., 2008).

Por outro lado, o presente estudo revela que as mães que referem uma tendência para atitudes de rejeição no seu comportamento parental percecionam os seus filhos como tendo um comportamento menos seguro e dificuldades de autorregulação emocional. Estes dados estão em consonância com as conclusões de vários estudos, segundo as quais os estilos educativos parentais que recorrem mais à rejeição estão associados a uma maior frequência de resultados desenvolvimentais negativos, designadamente a um apego mais inseguro (Brown \& Whiteside, 2008; Pereira, Canavarro, Cardoso, \& Mendonça, 2009). No mesmo sentido, os estudos que utilizam o EMBU para avaliar a perceção do comportamento parental também têm descrito uma associação entre o 
apego inseguro e níveis mais elevados de rejeição parental, e, em oposição, níveis mais baixos de suporte/afeto parental (Muris et al., 2000; Muris et al., 2003).

Foram desenvolvidas análises de regressão logística com o propósito de testar um modelo preditor da influência das dimensões afetivas (suporte emocional e rejeição) do estilo educativo materno na qualidade do apego da criança, tomando-se como variável dependente a pontuação global do PCV-M, que é um indicador da segurança do apego da criança. Foram definidas como variáveis independentes a perceção da mãe acerca do seu estilo educativo e o tipo de família, mas apenas as dimensões suporte emocional e rejeição foram incluídas nos modelos resultantes. A análise de regressão linear realizada permitiu ainda identificar que, de entre as dimensões da parentalidade avaliadas (suporte emocional, rejeição e tentativa de controlo), a rejeição materna é o melhor preditor da insegurança dos comportamentos de apego das crianças em idade escolar.

A principal ambição deste trabalho foi estudar a influência do estilo educativo materno na qualidade do apego da criança, considerando na análise a tipologia familiar. Um dos principais resultados do estudo indica que o tipo de família não é preditor dos comportamentos de apego das crianças em idade escolar, sugerindo que a tipologia familiar por si só não determina a qualidade do apego da criança. No mesmo sentido vão os resultados do estudo de Booth, Clarke-Stewart, McCartney, Owen e Vandell (2000), segundo os quais os estilos educativos parentais poderão ter um maior impato no percurso desenvolvimental das crianças, designadamente na qualidade do apego, em comparação com a estrutura familiar e com o estatuto marital dos pais.

A análise conjunta dos resultados permite constatar que as dimensões do estilo educativo materno que mais se relacionam com o comportamento de apego da criança, independentemente do tipo de família em estudo, são o suporte emocional e a rejeição, embora em sentidos opostos. Assim, como se tinha previsto inicialmente, parecem ser as dimensões afetivas do estilo educativo materno, suporte emocional e rejeição, as que mais influenciam na qualidade do apego da criança, reforçando a importância do estudo de outras variáveis que interferem na qualidade do apego provenientes da relação estabelecida entre pais e filho, para além da sensibilidade materna. Portanto, é corroborado que o estilo educativo que as mães utilizam na educação dos seus filhos pode ajudar a explicar a qualidade do apego da criança (Karavasilis et al., 2003; Kerns et al., 2001).

Estes resultados dão robustez à noção segundo a qual um estilo educativo materno caraterizado pela presença de suporte emocional promove comportamentos de apego seguros na criança, exactamente porque fomenta o estabelecimento de uma relação afetiva entre a criança e o seu progenitor com elevados níveis de sensibilidade, aceitação e expressão de afeto. Por seu lado, níveis ele- vados de rejeição contribuem para o desenvolvimento de um apego inseguro, possivelmente porque a criança se sente muito criticada, rejeitada e pouco amada o que, ao longo do tempo, poderá influenciar o desenvolvimento dos seus modelos internos dinâmicos.

Os dados desta pesquisa são reforçados por grande parte da literatura, que evidencia que os pais que estimulam o desenvolvimento da autonomia, que mantêm a monitorização e o controlo adequados e que, em paralelo, promovem o suporte e o afeto emocional à criança, fomentam a segurança do seu apego (e.g. Allen et al., 2003; de Minzi, 2006; Karavasilis et al., 2003). Em contraste, níveis mais elevados de rejeição parental, negligência ou controlo parental excessivo, ou níveis mais baixos de suporte/afeto parental estão associados a um apego inseguro (de Minzi, 2006; Muris et al., 2000; Muris et al., 2003; Roelofs et al., 2008).

Ainda que seja mais habitual o estudo da influência do comportamento parental na qualidade do apego da criança, tal como foi abordado no presente trabalho, não se deve esquecer a bidirecionalidade da interação entre estas duas variáveis. Neste sentido, é importante referir que, tanto o apego como a parentalidade são áreas complexas, sendo essencial a adopção de uma perspetiva ecológica, transacional e sistêmica que considere a multidimensionalidade dos fatores que influenciam a qualidade do apego da criança (e.g. van IJzendoorn \& Sagi-Schwartz, 2008).

Por fim, e a despeito do interesse empírico dos dados decorrentes do presente estudo, importa referir, como limitação metodológica, o número reduzido de crianças de famílias reconstituídas $(n=56)$ e a ausência de procedimentos de emparelhamento para este grupo de famílias, contrariamente ao que aconteceu com os grupos de famílias nucleares e monoparentais. De fato, tratando-se de uma subamostra tão específica, foi particularmente difícil obter um número de famílias reconstituídas equivalente ao número de famílias monoparentais e nucleares. Contudo, como os três grupos que compõem a amostra são equivalentes quanto às caraterísticas sociodemográficas (idade, gênero e ano de escolaridade da criança, número de irmãos e habilitações literárias da mãe), pode considerar-se que a validade externa do estudo, nomeadamente ao que à generalização dos resultados diz respeito, está assegurada.

Uma eventual limitação metodológica da escolha do EMBU-P para avaliar os estilos educativos parentais remete para a natureza dimensional do instrumento psicométrico. Isto é, reenvia ao fato de os seus itens refletirem a frequência com que ocorrem determinados padrões de comportamento concretos e não permitirem obter uma pontuação global do ambiente familiar ou das atitudes parentais. Ainda assim, optou-se pela abordagem dimensional dos estilos educativos parentais, já que esta abordagem possibilita a distinção do impacto que as diferentes dimensões do estilo educativo parental poderão ter no desenvolvimento da criança. 
Em função da importância atribuída na literatura aos processos de influência mútua pais-filhos, como, por exemplo, a socialização e o apego (processos recíprocos onde interagem as variáveis disposicionais de pais e de filhos, bem como o papel mediador de fatores relacionais e contextuais), a presente investigação procurou aproximar-se da perspetiva sistémica e transacional. Esta é a razão pela qual foi dada relevância ao estudo do tipo de família, bem como das variáveis comportamentais e desenvolvimentais e da influência que as dimensões do estilo educativo materno têm na qualidade do apego das crianças em idade escolar.

Em síntese, o presente trabalho, pelo seu enfoque simultâneo no estilo educativo materno e na qualidade do apego das crianças em idade escolar provenientes de diferentes tipos de família, constitui um contributo adicional e significativo às áreas da parentalidade e do apego. Constitui, ainda, uma abordagem pouco habitual que conjuga em simultâneo o estudo destes dois domínios importantes do desenvolvimento humano.

\section{Referências}

Allen, J. P., McElhaney, K. B., Land, D. J., Kuperminc, G. P., Moore, C. W., O'Beirne-Kelly, H., et al. (2003). A secure base in adolescence: Markers of attachment security in the mother-adolescent relationship. Child Development, 74, 292307.

Baumrind, D. (1989). Rearing competent children. In W. Damon (Ed.), Child development today and tomorrow (pp. 349-378). San Francisco, CA: Jossey-Bass.

Booth, C., Clarke-Stewart, K. A., McCartney, K., Owen, M. T., \& Vandell, D. L. (2000). Effects of parental separation and divorce on very young children. Journal of Family Psychology, 14, 304-326.

Bowlby, J. (1984). Attachment and loss: Vol 1. Attachment ( $2^{\text {nd }}$ ed.). London: Basic Books. (Original work published 1969)

Brown, A. M., \& Whiteside, S. P. (2008). Relations among perceived parental rearing behaviors, attachment style, and worry in anxious children. Anxiety Disorders, 22, 263-272.

Canavarro, M. C., \& Pereira, A. I. F. (2007). A avaliação dos estilos parentais educativos na perspectiva dos pais: A versão portuguesa do EMBU-P. Teoria, Investigação e Prática, 2, 271-286.

Cassidy, J. (1999). The nature of the child's ties. In J. Cassidy \& P. S. Shaver (Eds.), Handbook of attachment: Theory, research, and clinical applications (pp. 3-20). New York: Guilford Press.

Castro, L., Pablo, J., Gómez, J., Arrindell, W. A., \& Toro, J. (1997). Assessing rearing behavior from the perspective of the parents: A new form of the EMBU. Social Psychiatry and Psychiatric Epidemiology, 32, 230-235.

Darling, N., \& Steinberg, L. (1993). Parenting style as context: An integrative model. Psychological Bulletin, 113(3), 487 496.

de Minzi, M. C. R. (2006). Loneliness and depression in middle and late childhood: The relationship to attachment and parental styles. The Journal of Genetic Psychology, 167(2), 189-210.
Dias, P., Soares, I., \& Freire, T. (2002). Percepção materna do comportamento de vinculação da criança aos 6 anos: Construção de uma escala. Psicologia: Teoria, Investigação e Prática, 2, 335-347.

Dishion, T. J., \& McMahon, R. J. (1998). Parental monitoring and the prevention of child and adolescent problem behavior: A conceptual and empirical foundation. Clinical Child and Family Psychology Review, 1, 61-75.

Faber, A. J., \& Wittenborn, A. K. (2010). The role of attachment in children's adjustment to divorce and remarriage. Journal of Family Psychotherapy, 21(2), 89-104.

Farate, C., Pocinho, M., \& Machado, P. (2010). Repercussions of tobacco, alcohol and drugs on adolescent' health-Modalities of interaction and reciprocal influence. Saarbrucken, UK: Lambert Academic.

Feinberg, M. E., \& Kan, M. L. (2008). Establishing family foundations: Intervention effects on coparenting, parent/infant well-being, and parent-child relations. Journal of Family Psychology, 22(2), 253-263.

Grossmann, K. E., Grossmann, K., \& Kindler, H. (2005). Early care and the roots of attachment and partnership repre-sentations: The Bielefeld and Regensburg longitudinal studies. In K. E. Grossmann, K. Grossmann, \& E. Waters (Eds.), Attachment from infancy to adulthood: The major longitudinal studies (pp. 98-136). New York: Guilford Press.

Hetherington, E. M., Bridges, M., \& Insabella, G. M. (1998). What matters? What does not? Five perspectives on the association between marital transitions and children's adjustment. American Psychologist, 53, 167-184.

Kaczynski, K. J., Lindahl, K. M., Malik, N. M., \& Laurenceau, J.-P. (2006). Marital conflict, maternal and paternal parenting, and child adjustment: A test of mediation and moderation. Journal of Family Psychology, 20(2), 199-208.

Kalenkoski, C. M., Ribar, D. C., \& Statton, L. S. (2005). Parental child care in single-parents, cohabiting, and married-couple families: Time-diary evidence from the United Kingdom. Education and Health of Women and Children, 95(2), 194-198.

Karavasilis, L., Doyle, A. B., \& Markiewicz, D. (2003). Associations between parenting style and attachment to mother in middle childhood and adolescence. International Journal of Behavioral Development, 27(2), 153-164.

Kelly, J. B. (2007). Children's living arrangements following separation and divorce: Insights form empirical and clinical research. Family Process, 46, 35-52.

Kerns, K. A., Aspelmeier, J. E., Gentzler, A. L., \& Grabill, C. (2001). Parent-child attachment and monitoring in middle childhood. Journal of Family Psychology, 15, 69-81.

Kerns, K. A., Schlegelmilch, A., Morgan, T. A., \& Abraham, M. M. (2005). Assessing attachment in middle childhood. In K. A. Kerns \& R. A. Richardson (Eds.), Attachment in middle childhood (pp. 46-70). New York: Guilford Press.

Kilmann, P. R., Carranza, L. V., \& Vendemia, J. M. C. (2006). Recollections of parent characteristics and attachment patterns for college women of intact $v s$. non-intact families. Journal of Adolescence, 29, 89-102.

Lundberg, M., \& Andersson, P. (2000). Perception of the parental rearing when growing up in a single-parent home. Clinical Psychology \& Psychotherapy, 7(4), 275-278. 
Michiels, D., Grietens, H., Onghena, P., \& Kuppens, S. (2010) Perceptions of maternal and paternal attachment security in middle childhood: Links with positive parental affection and psychosocial adjustment. Early Child Development and Care, 180(1-2), 211-225.

Moura, O., \& Matos, P. M. (2008). Vinculação aos pais e conflito inter-parental em adolescentes. Psicologia (Lisboa), 22(1), 127-152.

Muris, P., Meesters, C., Merckelbach, H., \& Hülsenbeck, P. (2000). Worry in children is related to perceived parental rearing and attachment. Behaviour Research and Therapy, 38, 487-497.

Muris, P., Meesters, C., \& van der Berg, S. (2003). Internalizing and externalizing problems as correlates of self-reported attachment style and perceived parental rearing in normal adolescents. Journal of Child and Family Studies, 12(2), 171183.

Nair, H., \& Murray, A. D. (2005). Predictors of attachment security in preschool children from intact and divorced families. The Journal of Genetic Psychology, 166(3), 245-263.

Pereira, A. I. F., Canavarro, M. C., Cardoso, M. F., \& Mendonça, D. (2009). Patterns of parental rearing styles and child beha-viour problems among Portuguese school-aged children. Journal of Child and Family Studies, 18, 454-464.

Perris, C., Jacobsson, L., Lindstrom, H., von Knorring, L., \& Perris, H. (1980). Development of a new inventory assessing memories of parental rearing behaviour. Acta Psychiatrica Scandinavica, 61(4), 265-274.

Pettit, G., Bates, J., \& Dodge, K. (1997). Supportive parenting, ecological context, and children's adjustment: A seven-year longitudinal study. Child Development, 68, 908-923.

Roelofs, J., Meesters, C., \& Muris, P. (2008). Correlates of selfreported attachment (in)security in children: The role of parental romantic attachment status and rearing behaviors. Journal of Child and Family Studies, 17, 555-566.

Roelofs, J., Meesters, C., ter Huurne, M., Bamelis, L., \& Muris, P. (2006). On the links between attachment style, parental rearing behaviors, and internalizing and externalizing problems in non-clinical children. Journal of Child and Family Studies, 15(3), 331-344.

Roskam, I., Henry, M., Collin, B., \& Manil, P. (2008). Éducation parentale et non parentale: étude comparative auprès de dyades parent-infant en milieu familial et éducateur-enfant en milieu résidentiel. Enfance, 2, 158-176.

Schwartz, S. J., \& Finley, G. E. (2009). Mothering, fathering, and divorce: The influence of divorce on reports of and desires for maternal and paternal involvement. Family Court Review, 47(3), 506-522.

Soares, I. (2007). Desenvolvimento da teoria e da investigação da vinculação. In I. Soares (Ed.), Relações de vinculação ao longo do desenvolvimento: Teoria e avaliação (pp. 13-45). Braga, Portugal: Psiquilíbrios.

Thompson, R. A., \& Raikes, H. A. (2003). Toward the next quarter-century: Conceptual and methodological challenges for attachment theory. Development and Psychopathology, 15, 691-718.

Tippelt, G. G., \& Konig, L. (2007). Attachment representations in 6-year old children from one and two parent families in Germany. School Psychology International, 28, 313-330.

Trautmann-Villalba, P., Gschwendt, M., Schmidt, M. H., \& \& Laucht, M. (2006). Father-infant interaction patterns as precursors of children's later externalizing behavior problems: A longitudinal study over 11 years. European Archives of Psychiatry and Clinical Neuroscience, 256, 344-349.
Van IJzendoorn, M. H., \& Sagi-Schwartz, A. (2008). Crosscultural patterns of attachment: Universal and contextual dimensions. In J. Cassidy \& P. R. Shaver (Eds.), Handbook of attachment: Theory, research, and clinical applications ( $2^{\text {nd }}$ ed., pp. 880-905). New York: Guilford Press.

Woodward, L. J., Fergusson, D. M., \& Belsky, J. (2000). Timing of parental separations and attachment to parents in adolescence: Results from a prospective study from birth to 16 years. Journal of Marriage and the Family, 62, 162-174.
Recebido: 20/07/2011

$1^{a}$ revisão: $30 / 09 / 2011$

$2^{a}$ revisão: $28 / 11 / 2011$

Aceite final: $1 \% 12 / 2011$ 